\title{
Estrategia de promoción de salud para los docentes de Educación Básica
}

Enviado: 12 de febrero de 2021 / Aceptado: 19 de marzo de 2021 / Publicado: 31 de diciembre de 2021

\author{
YOANDRY RIVERO PADRÓN \\ Universidad Israel, Ecuador \\ yriverop@uisrael.edu.ec \\ (D) $\underline{0000-0002-1851-0899}$ \\ PATRICIA ALBUJA MARIÑO \\ Universidad Israel, Ecuador \\ palbuja@uisrael.edu.ec \\ (iD) $\underline{0000-0002-5033-7993}$ \\ BETTY PASTORA ALEJO \\ Universidad Israel, Ecuador \\ balejo@uisrael.edu.ec \\ (D) $0000-0002-9837-3264$
}

DOI 10.24310/IJNE.8.2021.11955

\section{RESUMEN}

Frente a las múltiples enfermedades que afectan al contexto social, como las infecciones contagiosas, el embarazo juvenil, entre otras, se requiere de la promoción para la salud, siendo esta un proceso que permite a los ciudadanos tener el control sobre ella y asumir comportamientos sanos en el buen vivir. Entonces, atribuyendo valor a esta idea, en el presente artículo se define como objetivo general proponer una estrategia de superación profesional, que prepare a los profesores de educación básica en la promoción y educación en salud, que debe cumplirse desde la misma organización de la actividad escolar, como parte inseparable del proceso de enseñanza-aprendizaje.

Los resultados del diagnóstico demuestran las falencias que presentan los profesores de este nivel escolar, debido a que desconocen las directrices del Ministerio de Educación en Ecuador, acerca de estas

\section{ABSTRACT}

Health promotion strategy for Basic Education teachers

In the face of multiple diseases that affect the social context such as contagious infections, teenage pregnancy, among others, health promotion is required, being a process that allows citizens to have control over their health and assume healthy behaviors in the good life. Therefore, attributing value to this idea in this article, the general objective is to propose a strategy for professional improvement that prepares basic education teachers in health promotion and education, which must be fulfilled from the very organization of the school activity as an inseparable part of the teaching-learning process.

Therefore, the results of the diagnostic show the shortcomings of teachers at this school level because they are unaware of the guidelines of the Ministry 
temáticas. Mientras tanto, las bibliografías consultadas avalan que la mayoría de las investigaciones están relacionadas con estrategias preventivas que no incluye un enfoque educativo desde las clases, a partir de una concepción integradora que facilite vincular los contenidos de su asignatura, con la cotidianidad que viven los discentes. Tal como se puede evidenciar en Giraldo, 2010; Gutiérrez, 2003; García, 2001.

Por otra parte, las orientaciones metodológicas se desarrollaron bajo el paradigma cuantitativo, a través de la investigación exploratoria con una muestra de 18 estudiantes de la Universidad Tecnológica Israel. En conclusión, se resalta la necesidad de capacitar al profesorado a fin de elevar sus conocimientos en el fomento de una cultura de salud integral en los discentes.

Palabras Clave: Estrategia, promoción, salud, educación básica. of Education in Ecuador on these issues. Meanwhile, the bibliographies consulted support that most of the research is related to preventive strategies that do not include an educational approach from the classrooms based on an integrative conception that facilitates linking the contents of their subject with the daily lives of the students. As can be seen in Giraldo, 2010; Gutiérrez, 2003; García, 2001.

On the other hand, the methodological orientations were developed under the quantitative paradigm through exploratory research with a sample of 18 students from the Israel University of Technology. In conclusion, it is evident the need to train the teaching staff in order to increase their knowledge for the promotion of an integral health culture in the students.

Keywords: Strategy, promotion, health, basic education.

\section{INTRODUCCIÓN}

La promoción para la salud conlleva a desarrollar conocimientos, actitudes, prácticas personales y sociales que inciden en los determinantes de la salud que fortalece hábitos, y estilos de vida en los individuos, hacia un nivel de bienestar óptimo. De este modo, se debe educar en la promoción, protección y prevención de la salud, en un proceso reflexivo permanente en las instituciones escolares y en todos los escenarios sociales que contribuya a la toma de conciencia individual, y colectiva en el saber bien vivir.

"La promoción de la salud permite que las personas tengan un mayor control de su propia salud. Abarca una amplia gama de intervenciones sociales y ambientales destinadas a beneficiar, proteger la salud, la calidad de vida individuales mediante la prevención y solución de las causas primordiales de los problemas de salud, y no centrándose únicamente en el tratamiento, y la curación” (Organización Mundial de la Salud, OMS, 2016, p.1).

Por consiguiente, la educación superior está llamada a atender los problemas inmediatos y prácticos que van incidiendo en la sociedad, anticipándose a su tiempo y promoviendo 
acciones en la posibilidad de construir un futuro sostenible y sustentable para las sociedades futuras. Entonces, es importante que, como respuesta a las diferentes problemáticas contemporáneas, incluyendo lo relacionado con la salud humana, se fortalezcan los circuitos de formación, y perfeccionamiento laboral, que es uno de los retos de la época, la formación y superación profesional del personal docente.

La superación del personal docente, sustentada en la concepción de la formación permanente, involucra comprender que la misma se extiende a lo largo de su vida laboral y responde a las necesidades personales, prioridades, y reclamos sociales que estimulan la adquisición, actualización, perfeccionamiento de los conocimientos, habilidades, capacidades, y actitudes inherentes a su actividad docente. La concepción de la escuela como escenario esencial, donde se orienta y desarrolla la superación de los docentes, exige que los profesores sean capaces de asumir los retos que impone el desarrollo social del presente.

La escuela como institución educativa está llamada a fortalecer la relación entre instrucción, educación y cultura, ya que ejerce una gran influencia en el individuo como partícipe de su sociedad; es el medio social encargado de unificar todos los factores sociales y para ello deben estar preparados los profesionales de la educación. Esta no sólo tiene el encargo social de transmitir conocimientos y desarrollar habilidades en los educandos, sino además contribuir a la formación de sentimientos, actitudes y valores que van conformando en el individuo una cultura general integral, de la cual forma parte la cultura en salud para propiciar una población más sana, y con adecuados estilos de vida.

En consecuencia, la promoción de la salud es el proceso que consiente a las personas incrementar el control sobre su salud para mejorar y modificar las condiciones sociales, ambientales y económicas que tienen impacto en los determinantes de la salud. Requiere de un proceso de capacitación (empowerment o empoderamiento) de personas y comunidades (Gutiérrez, 2003). En efecto, en la enseñanza actual se da una concepción pedagógica para el trabajo de promoción y educación para la salud que deben cumplirse desde la misma organización de la actividad escolar, con un enfoque eminentemente educativo, concebido como parte inseparable del proceso de enseñanza-aprendizaje en aras de lograr ciudadanos con estilos de vida cada vez más sanos.

De allí que, como iniciativa se puso en práctica las Escuelas Promotoras de la Salud (EPS) que generó un manual para la promoción de la salud, que hace énfasis en esta, siendo un derecho universal donde se da la importancia de la participación de los niños y las niñas, la comunidad en general, a modo de un proceso integrado de construcción de la ciudadanía (Red Ecuatoriana de Escuelas Promotoras de la Salud auspiciadas por el Ministerio de Educación, 2008). 
Por eso, la enseñanza primaria y secundaria, requiere de cambios significativos en la labor de los profesores, pues ellos asumen roles diferentes de acuerdo con la posición que ocupan en el sistema de influencias pedagógicas. El papel educador del docente en esta enseñanza da continuidad a todo el trabajo desarrollado en los diferentes niveles educacionales que le han precedido al estudiante donde los docentes de educación pueden planificar situaciones de aprendizaje, que a partir de los propios contenidos que se abordan en las clases, fortalezcan hábitos de vida saludables en los discentes.

Es importante que quienes imparten estos niveles, estén bien preparados en el proceder para hacerlo implicando el currículo de su asignatura. Por lo tanto, en la construcción de una cultura por la salud y la vida, se propuso lograr que todas las escuelas, como espacio potenciador en sus actividades programáticas, coadyuven en la promoción de la salud como un pilar fundamental para el desarrollo armónico de las colectividades (Ministerio de Educación del Ecuador, 2003). Asimismo, a nivel internacional se creó el comité de expertos sobre educación y promoción de la salud escolar.

Asimismo, se inicia el fortalecimiento de las acciones de salud a través de las escuelas, que son difundidas en varios foros y congresos internacionales (Organización Mundial de la Salud, 1995). Así pues, el término promoción de la salud fue propuesto por Henry E. Sigerist en el año 1945, cuando definió las cuatro grandes tareas de la medicina: promoción de la salud; prevención de riesgos y enfermedades; curación y rehabilitación del enfermo; la promoción como concepto de salud, que comienza a abordarse en la declaración de Alma Ata, donde se promulgo como meta fin la salud para todos. En dicha declaración se reconoce formalmente el enfoque de cuidados primarios de salud como estructura para el mejoramiento de la salud, en los habitantes del planeta (OMS, 1978).

Posteriormente, el 21 de noviembre de 1986, en Ottawa, Canadá, se lleva a cabo la primera conferencia internacional; el objetivo estaba dirigido a la implementación, por parte de los países industrializados, de los principios sostenidos en la declaración de Alma Ata: resumir directrices que proporcionen salud para todos los habitantes del planeta terrenal. En corolario, se redactó la Carta de Ottawa, donde se define que la promoción de salud debe proporcionar a los pueblos, los medios necesarios para mejorar su salud y ejercer un mayor control sobre esta (Citado en Díaz, et al. 2012).

Entre las principales experiencias, se destacan las escuelas promotoras de la salud apoyadas por el Municipio del Distrito Metropolitano de Quito; Programa de Espacios saludables en Loja y otras directamente desde el Ministerio de Educación, por medio de la División Nacional 
de Educación para la Salud. (Red Ecuatoriana de Escuelas Promotoras de la Salud, et al.). De esta manera, la salud se contempla como un recurso para la vida cotidiana; adicional a esto, la salud es un concepto positivo que enfatiza conseguir convivencias saludables hacia lo colectivo y personales, junto con capacidades físicas (Giraldo, 2010).

"El Estado promoverá una cultura por la salud y la vida con énfasis en la educación alimentaria y nutricional de madres y niños, en la salud sexual y reproductiva mediante la participación de la sociedad, y la colaboración de los medios de comunicación social" (Constitución Política de la República del Ecuador, 1998, Art. 43, acápite 2do, p. 15).

Se resalta la relevancia que tiene la promoción y educación para la salud en las escuelas en los primeros niveles de educación básica, donde el rol del educador, como promotor de la salud, es una figura de referencia significativa en el proceso formativo del educando, debido a que se convierte en un modelo a seguir en los estudiantes y también en el asesoramiento de la familia y la comunidad en general, cumpliendo con la gestión social que coadyuva en la mejora de las condiciones socio-culturales de cada localidad.

No obstante, a pesar de los avances de las ciencias y las tecnologías, la buena salud en la colectividad no se ha incrementado, ya que existen múltiples problemas sociales, económicos y personales que condicionan y afectan a miles de personas en el ámbito mundial, los cuales se pueden observar en el contexto social actual donde las principales enfermedades infectocontagiosas constituyen un problema de salud que impactan a la colectividad en general.

Por tal motivo, se puede indicar como ejemplo que, a nivel mundial más de 29 millones de niños menores de 5 años, sufren desnutrición aguda o grave con secuelas negativas en la vida de un niño que influye en un menor rendimiento y asistencia a la escuela (Fondo de las Naciones Unidas para la Infancia, UNICEF, 2015). De la misma forma, la afectación por el COVID- 19, con una tendencia de aceleración de su propagación y virulencia, requiere del asesoramiento a los países y a las personas en cuanto a las medidas para proteger la salud y prevenir el incremento de más brote, lo que está colaborando estrechamente con expertos mundiales, gobiernos y asociados para ampliar rápidamente los conocimientos científicos en este nuevo virus (OMS, 2019).

\section{Conviene puntualizar que:}

Los retos en el ámbito de la salud están dedicado al análisis de los principales factores causales de problemas de salud, los que representan los mayores desafíos y la mayor carga para la población de cada país o región. Estos retos y problemas se analizan en tres temas convergentes: los problemas de salud que se consideran cruciales debido a su gravedad, alta prevalencia, costos asociados o impacto a largo 
plazo en la salud de la población; los determinantes ambientales y sociales que están fuera del control directo o exclusivo del sector de la salud; y los obstáculos que se derivan de la falta de preparación de muchos sistemas de salud para hacer frente a los retos planteados por los problemas de salud y sus determinantes (Organización Panamericana de la salud, OPS, 2015, p. 1).

Cabe señalar que, en el caso específico de Ecuador, la mayoría de las investigaciones realizadas están vinculadas con estrategias preventivas fundamentalmente, dirigidas a las enseñanzas primarias y secundarias (Sexto, 2004; Del Río, 2007). Mientras tanto, los autores no pudieron constatar investigaciones de promoción para la salud, dentro de un enfoque educativo desde las clases. Situación que reafirma la importancia de comenzar en las instituciones escolares un plan de capacitación dirigido a los profesores de Educación Básica, con la finalidad de que desarrollen conocimientos y habilidades para vincular los contenidos de la asignatura que imparten en el nivel respectivo.

Por otra parte, las diferentes actividades educativas que realiza la escuela potencian el tratamiento a los temas de salud escolar; sin embargo, todos los profesores necesitan superarse que les permita desde la función que realizan, asumir correctamente su rol y para ello, se impone elevar la calidad en el desempeño profesional pedagógico con el propósito de lograr que se conviertan en promotores de salud, por medio de un proceso de capacitación permanente con un enfoque integrado en la reconstrucción de una nueva ciudadanía, en un estilo de vida más sano en el saber buen vivir.

El análisis antes realizado accede a determinar una contradicción dada por las exigencias planteadas desde el currículo de la Educación Básica, en función de la promoción y educación para la salud y la falta de superación teórico-metodológica de estos docentes para su dirección acertada en la práctica. En efecto, se propone una estrategia de superación profesional, que prepara a los docentes que imparten la materia, en cuanto a la promoción y educación para la salud, a partir de la combinación de las diferentes formas, teniendo en cuenta una planificación flexible y la comunicación dialógica para la socialización progresiva de los saberes.

El presente estudio muestra los fundamentos teóricos y metodológicos esenciales que sustentan la necesidad de la superación posgraduada del profesor de Educación Básica, en cuanto a la promoción y educación para la salud desde sus clases, lo que es avalado por una revisión bibliográfica actualizada en relación a la temática, que ofrece argumentos teóricos y prácticos que fundamentan la exigencia de contemplar en la superación del profesor de la Educación Básica. Esta realidad resalta la importancia de la necesidad de enseñar a la población en la promoción, y educación para la salud, especialmente en los espacios escolares dentro de sus 
clases diarias como un eje transversal que permee todos los contenidos de las asignaturas que contribuya a elevar el nivel de conocimiento a la vez tomen conciencia en el control de su propia salud y fomente otros estilos de vida.

\section{METODOLOGÍA}

La presente investigación toma en cuenta el enfoque cuantitativo que, es un proceso secuencial y probatorio que utiliza la recolección de datos para probar hipótesis con base en la medición numérica, y en el análisis estadístico que conduce a la revisión de la literatura que genera la construcción de un marco teórico referencial, para la solución de problemas o en la producción de nuevos conocimientos (Hernández, et al. 2014, p. 37).

Por consiguiente, el estudio se realizó en la Universidad Tecnológica Israel del Distrito Metropolitano de Quito, a través de la investigación exploratoria, con una población de 30 estudiantes de las carreras de Ciencias de la Educación: 13 de primer semestre y 17 del Nivel Ajuste Curricular. Por ello, se considera un muestreo estratificado de 18 estudiantes a quienes se les aplico una encuesta. De esta manera, la investigación exploratoria "es aquella que se efectúa sobre un tema u objeto desconocido o poco estudiado, por lo que sus resultados constituyen una visión aproximada de dicho objeto, es decir, un nivel superficial de conocimientos" (Arias, 2006, p. 24).

Del mismo modo, se creyó pertinente valerse del diseño documental, momento en el cual los investigadores asumieron que es un proceso basado en la búsqueda, recuperación, análisis, crítica e interpretación de la información que se obtiene en fuentes impresas o electrónicas para aportar nuevas ideas (Arias, et al. p. 28). Como referente a este criterio, se consultaron diversos autores que generaron sus aportes en el plano de la teoría, referente a la temática abordada, con la finalidad de demostrar la validez del análisis del contenido; técnica que sirve para describir sistemáticamente la información que permitieron plantear los siguientes objetivos de la investigación:

- Identificar el conocimiento de los maestros de educación primaria sobre el tratamiento curricular de la promoción y educación para la salud.

- Describir la importancia que tiene el rol del profesor como promotor de la salud en sus educandos, a partir de los contenidos de la asignatura que imparte.

- Establecer la necesidad de capacitación para los profesores en los diferentes temas disciplinares de la salud. 
Entonces, el objetivo general se centra en:

- Proponer una estrategia de superación profesional que prepare a los maestros de Educación Básica para la promoción y educación de la salud.

\section{RESULTADOS}

A continuación, se presentan los resultados del diagnóstico de esta investigación, que avalan la necesidad de proponer una estrategia de superación profesional que prepare a los maestros de Educación Básica en la promoción y educación para la salud, con la finalidad de que impartan desde sus clases diarias conocimientos que puedan servir al alumnado en el mejoramiento de su calidad de vida y en la contribución de ciudades más saludables en el saber buen vivir.

- De los 18 estudiantes encuestados, el 66,7 \% trabaja en colegios impartiendo materias en la Educación Básica mientras que, el 33,3 \% labora en sectores que no son educativos, pero estudian dicha carrera.

- El 61,1 \% (11 encuestados) plantean no conocer las directrices del Ministerio de Educación en Ecuador sobre el tratamiento curricular de la promoción y educación para la salud.

- $\quad$ El 77,8 \% (14 encuestados) desconocen los proyectos nacionales y territoriales que den tratamiento a la promoción y educación para la salud desde las aulas.

- El $100 \%$, consideran que el profesor, es un ente importante en la promoción y educación para la salud de los educandos.

- El $100 \%$, están de acuerdo como docente en capacitarse a modo de promotor de salud para poder tratar estos temas desde lo curricular con sus estudiantes.

- De los aspectos específicos de la promoción y educación para la salud que se relacionan son considerados por los encuestados deben ser incluidos en la preparación de los futuros maestros los siguientes:

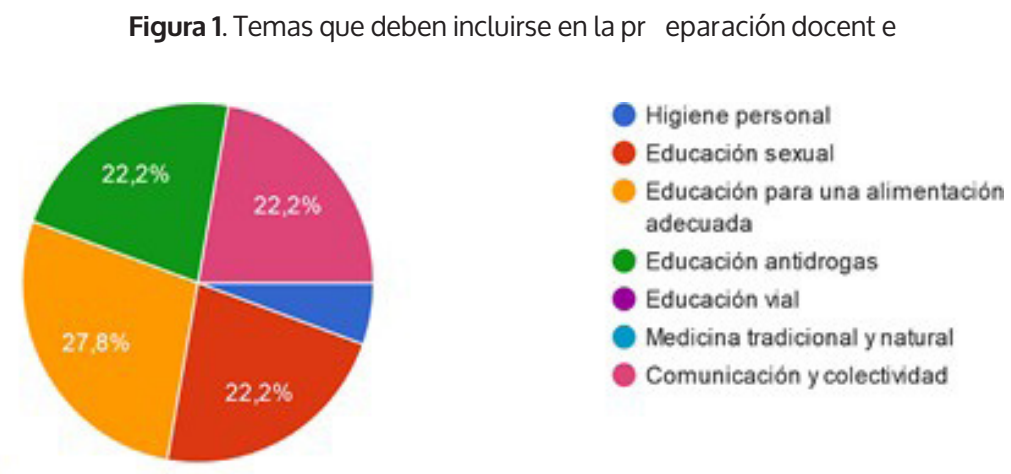


- Un $100 \%$, de los encuestados necesitan prepararse para poder trabajar con los estudiantes en los siguientes temas:

Figura 2. Necesidades de pr eparación de los docent es

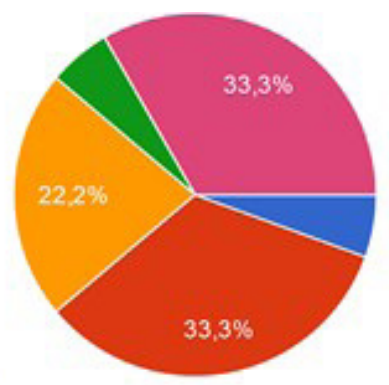

Comunicación y colectividad

- El 94,4\% (17 encuestados) indican que los contenidos de las materias que imparten pudieran utilizarse para que los discentes obtengan hábitos y estilos de vida saludables.

Los resultados demuestran las limitaciones que existen en los profesores tanto en el orden teórico como en el metodológico debido a que en su formación académica universitaria la Malla de Estudios de la Carrera de Educación Básica en cualquier universidad no ofrece un tratamiento adecuado del tema en la promoción para la salud en su formación como especialista de la educación básica y a las deficiencias que ha tenido la superación, situación que exige de una solución urgente por ser la promoción y educación para la salud un eje transversal de gran importancia en la Educación Básica.

Todo esto generó constatar en las investigaciones efectuadas en el ámbito internacional donde se han realizado aportes de vital importancia para los docentes esencialmente en la determinación de la concepción teórica de la promoción y educación para la salud en el contexto escolar, los conceptos básicos, los principios, las bases en que se sustenta y los documentos rectores que norman el trabajo de salud escolar en diferentespaíses.

Ahora bien, la implementación de proyectos en esta área, demanda de profesores con conocimientos, habilidades y metodologías apropiadas que logren resultados certeros para abordar temas relacionados con las ciencias y la salud. Por ello, la formación del profesorado en la actualidad ha de abordarse desde diferentes disciplinas que son necesarias en la actualización científica que permita disponer de informaciones recientes (Duta \& Canespecu, 2011).

Por lo tanto, "hay que trabajar para que... los problemas de salud estén integrado al proceso pedagógico y que parte de la pedagogía y la educación sea educar a los niños y jóvenes para que sean ciudadanos más saludables, plenos, para que tengan una noción de la vida cualitati- 
vamente superior" (Torres, et al. 1999, p. 2). Condición que exige la elaboración de estrategias de superación, direccionado a la promoción y educación para la salud.

Por otra parte, la superación profesional ha sido objeto de estudio de varios investigadores en los últimos años. Como consecuencia, las prácticas de formación presentan una escasa reflexión acerca del sentido de la enseñanza en los contextos actuales, en el marco de la reconfiguración del trabajo y de la identidad de los docentes y de las nuevas condiciones socio-culturales (Esteve, 2006).

Así que, mientras más preparado esté cada miembro de la sociedad, en especial los profesionales de la educación, más efectivo será el desarrollo de aquellos que se van incorporando a la vida social, por lo que la verdadera fuente de desarrollo de las cualidades de una persona la constituye la adquisición de la experiencia histórico-social acumulada.

En correspondencia con lo expresado se asume la superación profesional como un "conjunto de procesos de formación, que le posibilitan al graduado de los centros pedagógicos la adquisición y perfeccionamiento continuo de los conocimientos, habilidades básicas y especializadas, así como los valores ético-profesionales requeridos para un mejor desempeño de sus responsabilidades y funciones como docentes con vistas a su desarrollo general e integral" (García \& Addine, 2001, p. 17).

Por eso, se sostiene que la superación profesional comprende un largo proceso de formación, tanto en los estudios de grado, como de postgrado. Para ello, es importante definir tres procesos: los cursos o diplomados de actualización que se imparten desde la Universidad de Ciencias Pedagógicas; el trabajo metodológico que se desarrolla en la institución docente para lograr una retroalimentación inmediata desde la práctica pedagógica del colectivo y la autopreparación convenida a partir de las potencialidades, y deficiencias de la evaluación de su trabajo.

El segundo componente está referido a lo actitudinal, el cual se asume como la responsabilidad que tiene el profesor ante la labor de promoción y educación para la salud, que posibilite el desarrollo de una conducta responsable ante la vida (saber ser). Este presupone el compromiso y la responsabilidad ante la promoción y educación para salud, utilización de la autocrítica, la reflexión sobre su práctica educativa, en el manejo de una comunicación abierta, y flexible ante la labor de promoción, y educación de la salud, así como la utilización, y la 
confrontación de saberes, experiencias profesionales para enriquecer su superación respecto a la labor de promoción, y educación en salud.

Entonces, para que el maestro de educación básica pueda convertirse en un promotor de salud debe reunir los siguientes requisitos básicos:

- Poseer dominio de los temas de salud directrices del Ministerio de Educación que trabajará en la clase, así como la metodología de su asignatura.

- Actuar como modelo que proyecte una actitud de líder.

- Establecer buenas relaciones entre sus estudiantes y dentro del grupo escolar, manteniendo una actitud de respeto y autenticidad con el grupo para promover una comunicación abierta, atractiva y elocuente.

- Ser buen observador. Dirigir la atención y la mirada a todos los miembros del grupo, atender sus individualidades y las preocupaciones del colectivo de los estudiantes.

- Orientar y convencer, nunca imponer. Condición que le permite eliminar sus sesgos cognitivos en cuantos a los juicios y prejuicios personales.

- Manejar información relevante y las temáticas del programa, manteniéndose actualizado en el contenido que imparte.

El tercer componente, es el de actuación pedagógica, que se concibe como la manera en que el docente da solución a los problemas que se presentan en la práctica pedagógica, de forma creativa, mediante el cumplimiento de acciones estrechamente relacionadas con el propósito de contribuir a la formación de la personalidad apta para enfrentar la vida (saber hacer); en este, los docentes deben tener presente la identificación de los problemas fundamentales de la práctica pedagógica en torno a la promoción y educación para la salud.

De allí que, la formación de los profesores debe ser pensada en relación con el cambio educativo y que tome en consideración la lógica en una reflexión permanente (Vezub, 2005). De modo que, la estrategia de superación que se describe en esta investigación se organiza para su desarrollo teniendo en cuenta los siguientes pasos metodológicos. Estos elementos, desarrollados en 5 momentos, quedan gráficamente detallados en la Figura 3, los cuales permiten transitar por la consolidación teórica de la estrategia, la aplicabilidad y la valoración de la efectividad de la misma (Castillo \& Rodríguez, 2007, p. 4). 
Figura 3. Pasos de la estr ategia de super ación

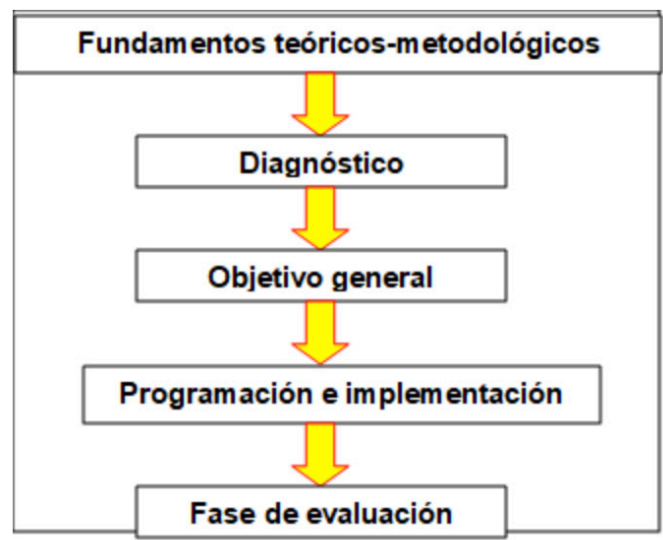

Por ello, los métodos a utilizar serán aquellos que estimulen la actividad productiva, el intercambio de experiencias y el debate de vivencias entre profesores; se propone la utilización de la elaboración conjunta, la enseñanza problémica y el trabajo independiente, entre otros. Igualmente, se distribuye el tiempo a partir de lo reglamentado en el ejercicio docente de profesor de Educación Básica para la superación profesional, dedicándose en el curso de superación el mayor número de horas a la autosuperación y fortaleciendo el trabajo metodológico, previsto su ejecución en un tiemplo establecido de dos meses; la realización de una adecuada planificación de los componentes didácticos de la asignatura en función de la promoción y educación para la salud. Manejo de otros medios didácticos como láminas, recortes de revistas o periódicos, las tecnologías de la información y la comunicación.

No obstante, la utilización del término estrategia, en el ámbito de las Ciencias Pedagógicas, comenzó aproximadamente a partir de 1960, coincidiendo con el comienzo del desarrollo de investigaciones dirigidas a describir indicadores relacionados con la calidad de la educación.

“(...) Toda estrategia transita por una fase de obtención de información que puede tener carácter diagnóstico; una fase de utilización de información y una fase de evaluación de esa información, además como su nombre lo indica, debe tener un margen para ir redirigiendo las acciones" (Ruiz, citado en Castillo, 2007, p. 151).

También, la estrategia se refiere a:

“...La dirección pedagógica de la transformación de un objeto desde su estado real hasta un estado deseado. Presupone, por tanto, partir de un diagnóstico en el que se evidencia un problema y la proyección y ejecución de sistemas de acciones intermedias, progresivas y coherentes que permitan alcanzar de forma paulatina los objetivos propuestos” (Pérez, 2003, p. 9). 
Las múltiples conceptualizaciones realizadas en torno a este término, hacen reflexionar acerca de la diversidad de criterios en vinculación a la definición de estrategia, distinguiéndose como aspecto significativo el hecho de reconocer en ella, la existencia de un componente intervencionista, expresado en el sistema de acciones, y el reconocimiento del diagnóstico inicial como punto de partida para lograr el estado deseado.

Sobre la base de la investigación realizada y abordando el tema desde una perspectiva integradora, se asume el criterio de reconocer la estrategia como una vía de solución para dar respuesta a los problemas relacionados con la superación que debe satisfacer las necesidades desde el punto de vista teórico y metodológico a partir del diagnóstico de las potencialidades y carencias de los docentes.

Las acciones que la conforman serán controladas y evaluadas sistemáticamente para transformar la realidad existente comenzando en un estado real a uno deseado que tome en cuenta una metodología formativa con contenidos de la pedagogía activa que, es importante utilizar técnicas y recursos didácticos para un trabajo de promoción de salud que ayude a analizar, y reflexionar sobre las situaciones o problemas actuales de la sociedad.

Por ello, la estrategia de promoción de la salud y prevención propone el desarrollo progresivo de intervenciones dirigidas a ganar salud y a prevenir las enfermedades, las lesiones y la discapacidad. Es una iniciativa que se desarrolla en el marco del Plan de Implementación de la Estrategia para el Abordaje de la Cronicidad en el Sistema Nacional de Salud (SNS, Ministerio de Sanidad, Servicios Sociales e Igualdad, 2014, p. 13).

En el caso particular de esta investigación, la estrategia que se propone posee las siguientes características:

- Flexible: porque puede sufrir cambios a partir de su implementación en la práctica pedagógica y la aplicación en diferentes contextos.

- Objetiva: implica que las diferentes etapas que se incluyen logren admitir acciones que parten de los resultados reales del diagnóstico de los profesores de educación básica para dirigir la promoción y educación en salud desde las problemáticas existentes en el contexto de actuación de los estudiantes.

- Sistémica: genera diferentes etapas que la conforman y las acciones manifiestan relaciones entre sí, que permiten la superación de los profesores para dirigir la promoción y educación en salud. 
- Problematizadora: en todas las acciones de superación de los profesores se logra la implicación de estos en la determinación y en la solución de problemas a partir de la búsqueda de información favoreciendo la creatividad en el plano metodológico para enfrentar el trabajo educativo de salud.

- Actualizada: el diseño de sus acciones estará en correspondencia con las orientaciones dadas por el Ministerio de Educación Superior del Ecuador con relación a la concepción del trabajo de promoción y educación para la salud en el ámbito escolar ecuatoriano y la estrategia responde a las condiciones, necesidades y exigencias en la dirección de la promoción y educación en salud tanto de los profesores de Educación Básica como del resto de los profesores que imparten su enseñanza en el sistema educativo básico, medio y de bachillerato.

- Formativa: en cada una de las acciones diseñadas se precisa con claridad qué hacer, cómo hacerlo, para qué y cómo controlarlo, lo cual los coloca en condiciones de asumir una posición de éxito y una actitud transformadora en relación con la labor de promoción, y educación para la salud que realiza. Por otro lado, se asumen como formas organizativas fundamentales para el desarrollo de las acciones de la estrategia: el curso, la autosuperación, la conferencia-taller y el trabajo metodológico.

El curso, está dirigido a la formación básica de los profesores, a partir de la organización de un conjunto de contenidos con el propósito de complementar y actualizar sus conocimientos, permitiendo la transformación del estado de insatisfacciones, que en cuanto al tratamiento en sus clases de la promoción y educación para la salud se presentan.

La autosuperación, posibilita a los profesores cumplir con las tareas orientadas en las diferentes formas organizativas de superación que hayan sido determinadas, que admite la retroalimentación de los resultados que se van obteniendo.

La conferencia-taller, facilita a partir de los conocimientos presentados por un informe o ponencia el intercambio y la interacción profesional sobre lo que se analiza, para la confrontación de ideas, juicios y opiniones, el ejercicio de la crítica, así como la adquisición de conocimientos adquiridos en procesos de investigación. Por último, el trabajo metodológico que contribuya a la preparación del maestro en la promoción y educación para la salud en los diferentes contenidos a impartir. 


\section{DISCUSIÓN}

Con relación a los resultados del diagnóstico y los referentes teóricos consultados en la investigación, se demuestra que existe la necesidad de capacitar por medio de la estrategia de superación teórico-metodológica a los maestros de Educación Básica en la promoción y educación para la saluddesde el inicio de sus clases en una concepción integradora a fin de que relacionen los contenidos de la asignatura con la cotidianidad que viven sus discentes.

La promoción de la salud es un tema que cobra vigencia en la actualidad, en razón a que se constituye en una estrategia básica para la adquisición y el desarrollo de aptitudes o habilidades personales que conlleva a cambios de comportamiento relacionados con la salud y al fomento de estilos de vida saludables, así como contribuir en el mejoramiento de la calidad de vida de las personas que conforman una sociedad, y en consecuencia, se logra la disminución en el coste económico del proceso salud- enfermedad y el deterioro ambiental (Giraldo, et al).

Del mismo modo, permite optar por estilos de vida saludables, tomar decisiones acertadas en un saber hacer para que tenga un mayor control de su propia salud, proteger el medio ambiente y asumir acciones en la promoción sanitaria en una corresponsabilidad compartida que contribuya al fomento la calidad de vida, en derivación, ciudades saludables en la cohabitación armoniosa, placentera y en el buen vivir.

Por tal motivo, la promoción de la Salud retoma las características y experiencias individuales, además de la valoración de las creencias en salud, en razón a que estas últimas son determinantes a la hora de decidir y asumir un comportamiento saludable o de riesgo para la salud, debido a su alto nivel de interiorización y la manera de ver la realidad que lo rodea (Pender, 2000, p. 38).

Se requiere un cambio de actitud por parte de la población en general para poner en práctica estilos de vida sanos en pro de mejorar la salud propia que consienta disminuir las conductas de riesgo en una modificación de los patrones adquiridos en su hogar, con los mismos pares y la comunidad inmediata que trascienda directa e indirectamente en el comportamiento saludable donde el maestro de Educación Básica debe asumir su rol protagónico en la sociedad actual.

\section{CONCLUSIONES}

Los grandes cambios que está viviendo la sociedad contemporánea requieren de una preparación continua y permanente de la salud como un derecho fundamental de toda persona. Por 
ende, el maestros de Educación Básica en el ejercicio profesional tiene la responsabilidad de promocionar estrategias en el desarrollo de sus clases para la promoción de la salud desde un enfoque integrador que permita en los estudiantes mejorar su calidad de vida a la vez que genere una convivencia en acciones virtuosas y saludables con la intención de promover un ambiente sano, positivo y sin violencia que se logrará a partir de un proceso educativo, reflexivo y participativo de la ciudadanía.

Por eso, la educación para la salud con un enfoque integral implica:

El desarrollo de procesos pedagógicos y metodológicos que orienten hacia la formación de aptitudes y habilidades para la vida, valores, conocimientos y prácticas de convivencia ciudadana y participativos en salud (Red Ecuatoriana de Escuelas Promotoras de la Salud, et al. p. 12). Tales cambios se alcanzarán si se afianzan los conocimientos que trascenderán en una nueva apertura cognitiva, actitudinal y social para la incorporación de prácticas saludables disminuyendo las conductas de riesgos y en ciudades armoniosas.

En efecto, la promoción de la salud, es un requerimiento urgente que debe llevarse a la práctica para lograr una sociedad sostenible y sustentable en el tiempo presente, y futuro que involucra un gran protagonismo de los docentes especialmente de la Educación Básica ya que en esos primeros años el niño o la niña puede adquirir otros estilos de vida que le consiente vivir funcionalmente desde una postura saludable y en integración con la sociedad a la cual pertenece. Esto se obtendrá afianzando los conocimientos que determinan en gran medida la adopción de prácticas saludables, puesto que aprueban a las personas tomar decisiones informadas y optar por estilos sanos o de riesgo frente al cuidado de su salud (Osorio, et al. 2010).

Es por ello, que los docentes en un profundo cambio de su manera de direccionar su práctica educativa enseñen al alumnado nuevos estilos de vida en nutrición sana, en actividades de recreación, el reconocimiento y la valoración de sí mismo en un nivel de auto estima elevado, la higiene personal, la salud sexual, y reproductiva, salud bucal, la comunicación asertiva que contribuya en su crecimiento físico, mental y de la personalidad.

Aunado a que les facilite ser promotor para la salud con conocimientos solidos que ayuden a los estudiantes a potenciar nuevas habilidades y capacidades con la finalidad de modificar las condiciones de vida tanto individual, y colectivo al cual pertenece que disminuye los determinantes de la salud que afectan a todo el estrato social en el ámbito mundial.

En definitiva, existe la necesidad de intervenir en el proceso de preparación de los profesores de Educación Básica en temas relacionados con la promoción y educación en salud. Una 
preparación pedagógica interactiva mediante la capacitación permanente y continua a partir de la combinación organizativa y planificada que garantice una secuencia flexible hacia un cambio profundo de su actuar docente en el aprovechamiento de todas las potencialidades de la escuela y de sus discípulos.

\section{REFERENCIAS BIBLIOGRÁFICAS}

Álvarez, L., Rodríguez, A., \& Sanabria, G. (2009). Salud sexual y reproductiva en adolescentes cubanos. Ciudad de la Habana: Ediciones abril.

Arias, F. G. (2006). El proyecto de investigación. Introducción a la metodología científica. Caracas, Venezuela. Sexta Edición. Editorial: Episteme.

Castillo, M. (2007). Estrategia de superación para los profesores de preuniversitario en la dirección de la educación para la salud. ISP "Félix Varela", Ciego de Ávila.

Constitución Política de la República del Ecuador. (1998). Carta Magna. Ecuador. Art. 43, acápite 2do.

Contreras, S. E. (2013). Pensamiento y Gestión. Universidad del Norte. Colombia. https://www.redalyc. org/pdf/646/64629832007.pdf

Del Río, L. (2007). La organización higiénica del régimen de vida. Una necesidad para el perfeccionamiento del proceso docente educativo en la enseñanza preuniversitaria. Cienfuegos.

Díaz Brito, Yoimy; Pérez Rivero Jorge Luis; Báez Pupo Francisco; Conde Martín Marlene (2012). Generalidades sobre promoción y educación para la salud. Universidad de Ciencias Médicas "Carlos J. Finlay”. Camagüey, Cuba. II Hospital Provincial “Manuel Ascunce Domenech”. Camagüey, Cuba.

Duta, N. \& Canespecu, M (2011). Características de un buen profesor universitario. hacia un perfil docente basado en competencias. Universidad de Bucarest. http://www.ub.edu/congresice/actes/9 rev.pdf

Esteve, J. (2006). Identidad y desafíos de la condición docente: vocación, trabajo y profesión en el siglo XXI. Tenti Fanfani, E. (comp.). El oficio de docente: vocación, trabajo y profesión en el siglo XXI. Buenos Aires: OSDE- IIPE/UNESCO, 19-69. https://www.redalyc.org/pdf/567/56711102.pdf

Fondo de las Naciones Unidas para la Infancia (UNICEF, 2011). Datos y cifras clave sobre nutrición. https://www.unicef.org/media/62486/file/Estado-mundial-de-la-infancia-2019.pdf

García, G., \& Addine, F. (2001). La formación permanente de los docentes de la educación superior tecnológica. Un reto para la optimización del rol profesional pedagógico. https://repositorio.itb.edu.ec/ bitstream/123456789/847/1/Carlos \%20Rivera.pdf 
Giraldo, A., Toro, Y., Macías, A., Valencia, C., Palacio, S. (2010). La promoción de la salud como estrategia para el fomento de estilos de vida saludables. Revista hacia la promoción de la salud. Universidad de Caldas. Colombia. https://www.redalyc.org/pdf/3091/309126693010.pdf

Gutiérrez G, José J. (2003). Formación en promoción y educación para la salud. informe del grupo de trabajo de promoción de la salud a la comisión de salud pública del consejo interterritorial del sistema nacional de salud. Ministerio de Sanidad y Consumo. Secretaría General Técnica. Madrid. España.

Hernández, S. R., Fernández, C., \& Pilar, B. L. (2014). Metodología de la Investigación. Sexta Edición. México DF. McGrawHill. https://periodicooficial.jalisco.gob.mx/sites/periodicooficial.jalisco.gob. $\underline{\mathrm{mx} / \text { files/metodologia de la investigacion - roberto hernandez sampieri.pdf }}$

Ministerio de Educación del Ecuador. (2003). Escuelas promotoras de la salud. Gobierno Nacional de la República del Ecuador. https://bibliotecapromocion.msp.gob.ec/greenstone/collect/promocin/ index/assoc/HASH01c8.dir/doc.pdf

Ministerio de Sanidad, Servicios Sociales e Igualdad (2014). Informes, Estudios e Investigación. Estrategia de promoción de la salud y prevención en el SNS En el marco del abordaje de la cronicidad en el SNS Estrategia aprobada por el Consejo Interterritorial del Sistema Nacional de Salud el 18 de diciembre de 2013. https://www.mscbs.gob.es/profesionales/saludPublica/prevPromocion/Estrategia/docs/EstrategiaPromocionSaludyPrevencionSNS.pdf

Organización Mundial de la Salud (1978). Carta de Ottawa para la promoción de salud. https://apps. who.int/iris/bitstream/handle/10665/39244/9243541358.pdf;jsession id=DDD5696B12419DD87 B6B2A4305416EB0?sequence $=1$

Organización Mundial de la Salud (1995). ¿Qué es la promoción de la salud? https://www.who.int/features/qa/health-promotion/es/

Organización Mundial de la Salud (2016). Repaso de la salud mundial. https://instituciones.sld.cu/socu$\underline{\text { sap/2017/01/10/resumen-del-2016-repaso-de-la-salud-mundial-de-la-oms/ }}$

Organización Mundial de la Salud (2019). Brote de enfermedad por coronavirus (COVID-19). https:// www.who.int/es/emergencies/diseases/novel-coronavirus-2019?gclid=CjwKCAiAiML-BRAAEiwAuWVggvsq7GHH8zRMnxPyZZugSHuxkGVmmbFlys9XAwMkjn7zCjqxWGtguxoCU3cQAvD BwE

Organización Panamericana de la Salud(2015). Principales problemas y retos en el ámbito de la salud:Introducción. https://www.paho.org/salud-en-las-americas-2017/?post t es=introduccion\&lang=es 
Osorio, A. Giraldo; Toro R. María; Macías, Ladino; Garcés, Carlos; Rodríguez, Sebastián. (2010). La promoción de la salud como estrategia para el fomento de estilos de vida saludables. Universidad de Caldas. Colombia. https://www.redalyc.org/pdf/3091/309126693010.pdf

Pender N. J. (2010). Conferencia Taller dirigido a profesionales de enfermería y del área de salud. Modelo de Promoción de la Salud; Oct 2000. 32. Alarcón A, Vidal A, Neira

J. Salud. https://www.redalyc.org/pdf/3091/309126693010.pdf

Pérez, N. (2003). La capacidad. Tesis doctoral, Universidad "Carlos Rafael Rodríguez". Primo, M. a. (2001). Modelo básico para la superación del docente como maestro-investigador. La Habana.

Red Ecuatoriana de Escuelas Promotoras de la Salud auspiciadas por el Ministerio de Educación(2008). Manual de Escuela Promotoras de la Salud. Tercera edición. Quito. Ecuador.

Rodríguez, G. (2007). Estrategia pedagógica de preparación a los docentes de Ciencias Naturales en formación inicial intensiva para dirigir la educación para la salud.Tesis de doctorado. Ciego de Ávila. Cuba.

Sexto, N. (2004). Propuesta de estrategia educativa para la salud bucal de los estudiantes de primaria. Cienfuegos. Cuba.

Torres, M. A., Carvajal, C., Machado, A., Sánchez, M., González, A., Gómez, y. (1999). Programa Directro de Promoción y Educación para la salud en el Sistema Nacional de Educación. Molinos Trade.

Vezub, L. (2005b). El discurso de la capacitación docente. Una aproximación a las políticas de perfeccionamiento en la Provincia de Buenos Aires. Espacios en Blanco, Revista de Educación, 15, 211-242. 